\title{
Mapping the fragmentation of acetylene with femtosecond resolution pump probe at LCLS using 2,3 , and 4 particle coincidences
}

\author{
Chelsea E. Liekhus-Schmaltz ${ }^{1, *}$, Ian Tenney ${ }^{1}$, Timur Osipov², Philip H. Bucksbaum ${ }^{1,2}$, Vladimir S. Petrovic ${ }^{1}$, \\ and the AMO75113 collaboration [1] \\ ${ }^{1}$ Stanford University, 382 Via Pueblo Mall, Stanford, CA 94305-4060 \\ ${ }^{2}$ SLAC National Accelerator Laboratory, 2575 Sand Hill Road, Menlo Park, CA 94025 \\ *cliekhus@stanford.edu
}

\begin{abstract}
A three-layer delay line anode detector has been used in x-ray pump x-ray probe timeresolved measurement at LCLS. We used $\sim 10$ fs long pulses to initiate and probe ultrafast dynamics in the dication of acetylene. The dynamics are discerned from the temporal evolution of multi-particle coincidences.

OCIS codes: (320.7120) Ultrafast phenomena; (020.2649) Strong field laser physics
\end{abstract}

\section{Introduction}

The dissociative ionization of the acetylene dication has been the subject of this study in order to understand how the relaxation upon core ionization of a prototypical hydrocarbon occurs. There are four two-particle fragmentation pathways in the dication, two that involve deprotonation (P1) and (P2), a symmetric pathway that involves first breaking the bond between the two carbons (A), and a pathway through which acetylene first isomerizes to vinylidene $(\mathrm{V})$. The $(\mathrm{V})$ pathway is of particular interest because it involves proton migration.

The states associated with each of these pathways have been identified by T Osipov et. al. [2]. Synchrotron measurements put an upper bound of $60 \mathrm{fs}$ on the time in which proton migration occurs [3]. However, the theoretically predicted $\sim 50 \mathrm{kcal} / \mathrm{mol}$ isomerization barrier between acetylene and vinylidene in the ground state of the dication predicts a longer isomerization time $[4,5]$.

To probe this process further, we have conducted a time-resolved x-ray pump x-ray probe experiment at LCLS. We can completely resolve the energy and momentum of the ion fragments generated, and can therefore identify different decay channels. By monitoring how the different decay channels evolve in time, we have the opportunity to better understand each of the pathways.

\section{Experimental details}

We performed an x-ray pump x-ray probe experiment on both acetylene and deuterated acetylene $\left(\mathrm{C}_{2} \mathrm{D}_{2}\right)$ using $400 \mathrm{eV}$ (just above the K-edge of carbon) $\mathrm{x}$-ray pulses with duration of $\sim 10 \mathrm{fs}$. The pump pulse core-ionizes the sample. The Auger decay that follows impulsively excites states within the energy range that corresponds to the inverse of the core-hole lifetime ( $\sim 6 \mathrm{fs}$ in carbon) [2]. The ensuing dynamics are probed by the second $\mathrm{x}$-ray pulse that, after subsequent core ionization and Auger process, initiates Coulomb explosion in the tetracation. The delay between the pump and probe x-ray pulses was varied between 0 and $100 \mathrm{fs}$. Two methods were used to produce two nearly-identical x-ray pulses: the slotted spoiler method and the newly installed split and delay apparatus $[6,7]$.

The measurement was done in a molecular beam under the conditions where on average one molecule fragments per event. The ion fragments are detected using a newly commissioned delay line anode detector. This detector allows us to resolve both the position and arrival time of each ion fragment, yielding all three components of the momentum. In addition, this detector has 3 layers of delay lines, which increases the number of individual ions that can be resolved when they arrive at similar times and position, permitting us to detect multi-particle coincidences. Figure 1 illustrates the benefits of the three-layer detector compared to a two-layer one.

\section{Analysis}

The process of analyzing the data relies on the ability to identify "real" coincidences, i.e. combinations of ion fragments that result from the decomposition of the same acetylene molecule. This is achieved by applying constraints on each potential coincidence based on conservation of momentum and energy. A visualization of this is shown in Fig. 2 for the case of the (V) decay pathway after a single photon absorption. In addition to being able to observe 2 particle coincidences as shown in Fig. 2, initial results have shown that we can in fact resolve 3 and 4 particle coincidences. This permits probing the x-ray initiated ultrafast dynamics in great detail. 

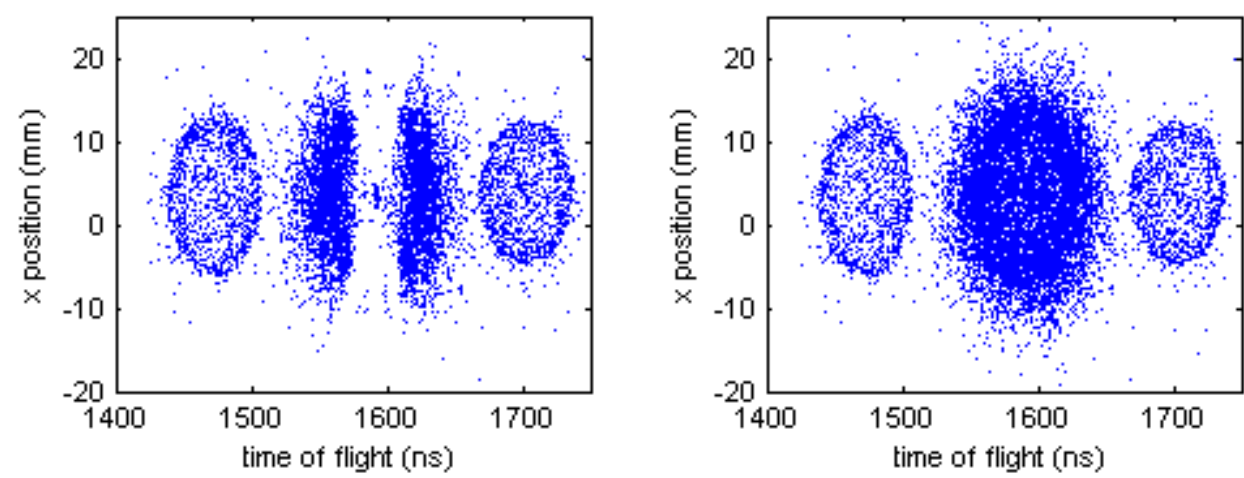

Fig. 1. To demonstrate the benefits of the three layer delay line anode detector (right) compared to a two layer one (left) for resolving ion fragments with similar arrival time and position, we plot the x position vs. time of flight for a subset of ions in two particle coincidences. The $\mathrm{CD}^{+} / \mathrm{CD}^{+}$pairs can be seen as the feature at $\sim 1600 \mathrm{~ns}$ (the features at $\sim 1470$ and $\sim 1700 \mathrm{~ns}$ are due to $\mathrm{C}^{+} / \mathrm{CD}_{2}{ }^{+}$coincidences). The events that are discarded due to the inability of the two-layer detector to resolve them result in a missing portion of the data on the left while the same portion of the data can be resolved with the redundancy provided by the three-layer detector on the right.

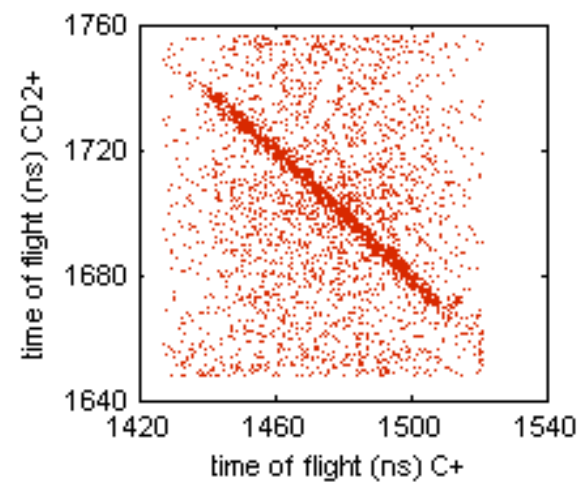

Fig. 2. To find coincidences corresponding to the isomerization pathway (V) after the $\mathrm{x}$-ray photon absorption, coincidences of $\mathrm{C}^{+}$and $\mathrm{CD}_{2}^{+}$ are found. For true coincidences the conservation of the momentum (along the time of flight axis in this case) results in the data lying on a line as shown above. Analysis of the temporal evolution of the multi-particle coincidences will provide the necessary information about the x-ray initiated change of the molecular geometry associated with this pathway.

\section{References}

[1] AMO75113 collaborators: Ali Belkacem, Nora Berrah, Rebecca Boll, Cedric Bomme, Christoph Bostedt, John David Bozek, Sebastian Carron, Ryan N. Coffee, Julien Devin, Benjamin Erk, Li Fang, Robert W. Field, Ken Ferguson, Lutz Foucar, Leszek J. Frasinski, James Michael Glownia, Markus Guehr, Andrei Kamalov, Jacek Krzywinski, Heng Li, Jonathan P. Marangos, Todd Martinez, Brian K. McFarland, Shungo Miyabe, Brendan F. Murphy, Adi Natan, Daniel Rolles, Artem Rudenko, Alvaro Sanchez, Marco Siano, Emma Simpson, Limor S. Spector, Michele Lee Swiggers, Daniel J. Walke, Song Wang, and Thorsten Weber

[2] T. Osipov, et. al., "Fragmentation pathways for selected electronic states of the acetylene dication," J. Phys. B 41, 091001 (2008).

[3] T. Osipov, et al, "Photoelectron-Photoion momentum spectroscopy as a clock for chemical rearrangements: Isomerization of the di-cation of acetylene to vinylidene configuration," PRL 90 (23), 233002 (2003).

[4] T. S. Zyubina, Y. A. Dyakov, S. H. Lin, A. D. Bandrauk, A. M. Mebel., "Theoretical study of isomerization and dissociation of acetylene dication in the ground and excited electronic states," J. Chem. Phys. 123, 134320 (2005).

[5] D. Duflot, J-M. Robbe, and J-P. Flammet, "Ab initio study of the acetylene and vinylidene dications fragmentation," J. Chem. Phys. 102 (1), 355 (1995).

[6] P. Emma, K. Bane, M. Cornacchia, Z. Huang, H. Schlarb, G. Stupakov, and D. Walz, "Femtosecond and subfemtosecond x-ray pulses from a self-amplified spontaneous-emission-based free-electron laser," PRL 92 (7), 074801 (2004).

[7] B. Murphy, J.C. Castagna, J. Bozek, and N. Berrah, "Mirror-based soft x-ray split-and-delay system for femtosecond pump-probe experiments at LCLS," in X-Ray Free-Electron Lasers: Beam Diagnostics, Beamline Instrumentation, and Applications, Stefan P. Moeller, Makina Yabashi, Stefan P. Hau-Riege. Vol. 8504 of SPIE Proceedings, (International Society for Optics and Photonics, Washington, 1955).

This work was carried out in the Stanford PULSE Institute through the support of the National Science Foundation. 